\title{
Call for Submissions / Appel à candidatures
}

\author{
Patterson Prize 2021
}

Award: \$30o USD and publication in Simone de Beauvoir Studies

Deadline: March 1, 2021

The Editorial Committee of Simone de Beauvoir Studies is pleased to announce the first annual Patterson Prize competition. The Patterson Prize is awarded to a work that demonstrates excellence in writing while embodying modes of thought and expression characteristic of Beauvoir's oeuvre. We especially invite submissions on topics relevant to Beauvoir's legacy such as gender and sexuality, race and culture, literature, existentialism, global politics, and others. For this international competition, the Patterson Prize Committee welcomes texts in English and French from all disciplines and genres of writing. Entries should be 8 ooo words or less inclusive of notes and references. For detailed information about eligibility and submission requirements, see the About/Downloads Tab at www.brill.com/sdbs.

\section{Prix Patterson 2021}

Prix: 300 \$ USD et la publication du texte récompensé dans la revue Date limite: $\mathbf{1}^{\mathrm{er}}$ mars 2021

Le comité éditorial de la revue Simone de Beauvoir Studies a le plaisir d'annoncer le lancement du premier concours annuel du Prix Patterson, qui est décerné à un texte s'inscrivant dans la lignée beauvoirienne tant par la qualité de son écriture que par la manière dont y sont traités des sujets liés à l'œuvre de l'autrice et philosophe: les études sur le genre (gender studies), la sexualité, le féminisme, les rapports interculturels, le postcolonialisme, le militantisme politique, l' existentialisme et la littérature. Le Prix Patterson est remis dans le cadre d'un concours international qui accueille des textes en anglais et en français issus de domaines de recherche et de genres littéraires variés. Les textes soumis doivent compter 8 ooo mots ou moins, y compris les notes et la bibliographie. Pour découvrir comment soumettre une candidature, nous vous invitons à consulter l'onglet «About/Downloads» de la page suivante: www .brill.com/sdbs. 